\title{
A comparison of two methods of logMAR visual acuity data scoring for statistical analysis
}

\author{
†OA Oduntan, *KP Mashige and **M Raliavhegwa-Makhado
}

$\dagger$, ${ }^{*}$ Discipline of Optometry, School of Physiotherapy, Sport Science and Optometry, Faculty of Health Sciences, University of KwaZulu-Natal, Private Bag X 54001, Durban, 4000 South Africa

\author{
**Department of Optometry, University of Limpopo, Private Bag X1106, Sovenga, 0727 South \\ Africa
}

Received 29 March 2009; revised version accepted 2 September 2009

\begin{abstract}
The purpose of this study was to compare two methods of logMAR visual acuity (VA) scoring. The two methods are referred to as letter scoring (method 1) and line scoring (method 2). The two methods were applied to VA data obtained from one hundred and forty $(N=140)$ children with oculocutaneous albinism. Descriptive, correlation and regression statistics were then used to analyze the data. Also, where applicable, the Bland and Altman analysis was used to compare sets of data from the two methods. The right and left eyes data were included in the study, but because the findings were similar in both eyes, only the results for the right eyes are presented in this paper. For method 1 , the mean unaided VA (mean UAOD1) $=0.39 \pm$ $0.15 \log M A R$. The mean aided (mean ADOD1) VA $=0.50 \pm 0.16 \log$ MAR. For method 2 , the mean unaided (mean UAOD2) VA $=0.71 \pm 0.15$ log-
\end{abstract}

\section{Introduction}

Snellen VA charts and notations are used extensively for the measurement of visual acuity (VA) for clinical, research and socio-legal purposes, but various deficiencies in the charts have been highlighted by various authors ${ }^{1,2}$. The (logMAR) method of acuity scaling devised by Bailey and Lovie ${ }^{1}$ is now considered to be more appropriate for scaling VA meas-
MAR, while the mean aided VA (mean ADOD2) = $0.60 \pm 0.16 \log M A R$. The range and mean values of the improvement in VA for both methods were the same. The unaided VAs (UAOD1, UAOD2) and aided (ADOD1, ADOD2) for methods 1 and 2 correlated negatively (Unaided, $r=-1, p<0.05$ ), (Aided, $r=-1, p<0.05$ ). The improvement in VA (differences between the unaided and aided VA values) (DOD1 and DOD2) were positively correlated $(r=+1, p<0.05)$. The Bland and Altman analyses showed that the VA improvement (unaided - aided VA values) (DOD1 and DOD2) were similar for the two methods. Findings indicated that only the improvement in VA could be compared when different scoring methods are used. Therefore the scoring method used in any VA research project should be stated in the publication so that appropriate comparisons could be made by other researchers.

Key words: logMAR acuity, Snellen acuity, visual acuity scoring methods, statistical analysis urements and has been used in the design of all recent VA charts ${ }^{1-6}$. LogMAR is an acronym for $\log _{10}$ of the minimum angle of resolution (MAR), where MAR is the width of the stroke or one-fifth of the angular subtense of the optotype. LogMAR values are presented in numerical format such as 1.0, 0.9, 0.8, 0.7 logMAR, thereby making data scoring and analysis easier.

For over a decade, the recording of acuities as logMAR notation has been advocated ${ }^{7}$. The major advan- 
tage of this notation especially for research purposes is the ability to measure and score low VA accurately, which can then be included in statistical analysis ${ }^{8}$. Another advantage is the regular progression of the optotype sizes from one line to the other in geometric format as each optotype on one line is 1.2589 times larger than the optotype on the next $\mathrm{t}^{1,2}$. This multiplier is the tenth root of ten, $\sqrt[10]{10}$ or $0.1 \log$ unit $^{1,2}$. These features permit inter-row interpolation of VA values. Since there is 0.1 logMAR unit difference between acuity lines of the logMAR charts, when each line has five optotypes, an interpolated logMAR score can be created by assigning 0.02 logMAR units for each optotype read correctly ${ }^{2}$.

In spite of the disadvantages of the Snellen chart and the advantages of the logMAR chart, the Snellen recording methods are still widely used in clinical practice and research. A recent study ${ }^{9}$ which investigated the different VA recording methods used in peer-reviewed ophthalmology clinical studies over the past decade found that, overall, $10 \%$ of the authors appeared to have converted Snellen acuity measurements to logMAR format rather than scoring directly as logMAR. The persistent use of Snellen notation, particularly in clinical practices is obviously due to the relative simplicity of the method compared to the logMAR system ${ }^{9}$.

The use of Snellen notation in recording VA does not preclude data so obtained from being converted to $\log$ MAR, provided that a logMAR chart has been used to collect the data. One method of doing this is to take the $\log _{10}$ of the reciprocal of the enumerated Snellen Fraction ${ }^{7,}$ 10. For example, if a patient read $6 / 10$ (Snellen), the reciprocal is $10 / 6=1.7$. Taking the $\log$ of this, $1.7 \log 10=0.23 \log$ MAR. This conversion can be done regardless of the denominator or numerator of the Snellen acuity ${ }^{10}$. There are cases, however, when letters missed in the best VA line or gained in the next line are recorded in the Snellen format as $6 / 18^{-2}$ or $6 / 18^{+2}$. In the logMAR principle, the number of letters missed or gained can be calculated and adjusted from the best VA line to arrive at a single logMAR VA value. There are two methods of doing this as discussed below.

Ferris et $a l^{2}$ indicated that the principle of interpolating logMAR score can be applied by assigning $0.02 \log$ MAR units for each letter read correctly in situations in which some letters on several lines are missed. By scoring 0.02 for each letter read correctly on the entire chart and adding these scores, a VA score can be created that is a single number and is a reasonable estimate of the logMAR score for that eye. The authors $^{2}$ gave this example: In a chart containing 5 letters per line, if all letters up to 6/7.5 (logMAR 0.1) are correctly read and in addition, three letters on the next line (6/6 line) (logMAR 0) are read, the VA value will be $1.06 \log$ MAR $(53 \times 0.02=1.06)$ or $(10 \times 0.1$ $+0.06=1.06$ ) since each line is $0.01 \log$ MAR). This method is frequently used for VA scoring for research purposes, but not used clinically because it simply measures the total number of letters read, not the size of the smallest line read.

According to Ferris et $a^{2}$ the interpolated logMAR method score can be applied by simply assigning 0.02 logMAR units for each letter read correctly on the chart and adjusting for the letters missed or gained on the best VA line. Using the above example: If all letters up to 6/7.5 (logMAR 0.1) are correctly read and in addition, three letters on the next line (6/6 line) (logMAR 0$)$ are read; an interpolated value of 0.04 $\operatorname{logMAR}$ (that is, $0.1-(3)(0.02)=0.04$ can be used to represent the VA. This method is used both clinically and for research purposes in scoring VA.

These two methods of acuity scoring have been used in different studies under different names, and so, it is sometimes difficult to know exactly which method is being used. For instance, the second method has been referred to as single letter scoring by Rosser et $a l^{10}$ and the authors devised a formula (logMAR $\mathrm{VA}=1.1-\mathrm{TCLV}$ ) for calculating the VA values, where TC is the total number of correctly read letters and $\mathrm{LV}$ is the logMAR value of each letter on the chart. For instance, if a subject read five lines $(6 / 24)$ on a logMAR chart having acuity lines from 6/60 (1.0 $\log M A R)$ to $6 / 3$ (-0.3 logMAR), the total number of letters read will be 25 . Since there are five letters per row on the chart, the value of each letter is 0.02 logMAR unit. The logMAR VA value $=1.1-(25 \times 0.02)$ $=0.6$, which is the logMAR acuity for that line on the chart. This formula applies, even when only a few letters are read on the best or smallest acuity line ${ }^{10}$.

For research purposes, the first method has been used by several authors $5,11,12$ and the second by Rosser et $a^{10}$. Although the two methods involve assigning 0.02 or any relevant logMAR unit value, depending on the number of letters per row of the chart 
employed; a casual observation shows that the two methods are different in their applications and acuity values. An important question is; when these two methods are applied independently to a large number of varying VA values, will the resultant acuity values differ? The aim of this study therefore, was to compare results obtained from these two methods when they are applied to the same sets of acuity data. The objectives of this study are (i) To determine whether there is any consistent association or relationship (positive or negative relationship) between VA values obtained using the two methods, (ii) To establish, if there is a statistically significant relationship between the VA values resulting from the two methods of VA scoring.

\section{Methodology}

Visual acuity data obtained from one hundred and forty $(N=140)$ children with oculocutaneous albinism in research towards a master's degree by Makhado (Raliavhegwa ${ }^{13}$ ) were used in this study. The data have not been published elsewhere and were considered useful for this study. A logMAR chart designed by Oduntan and Briggs ${ }^{5}$ was used for the VA measurements, but the values were recorded in Snellen format for example $6 / 18^{-2}$ or $6 / 24^{+2}$. The VA data were converted to logMAR format using the method 1 , which we referred to as letter scoring and method 2 , which we referred to as line scoring. The conversions were done as described below, using the same logMAR chart that was used to collect the data.

\section{Letter scoring (method 1)}

Each letter read by a subject was noted and multiplied by 0.02. Example: A patient correctly read all the letters from top up line to $(6 / 12)$ and two letters on the next (6/10) line (Snellen acuity 6/12+2). The logMAR VA can be calculated as $0.1(8)+(2)(0.02)$ $=0.84 \log M A R$. The formula: $\log$ MAR score $(0.1 \mathrm{NL}$ $+0.02 \mathrm{nl}$ ) where NL $=$ number of lines completely read and $\mathrm{nl}=$ number of additional letters read applies. For this method, the higher the logMAR VA score, the better the visual acuity.

\section{Line scoring (method 2)}

The logMAR value of the best line read was noted and the number of letters read in the next row was multiplied by 0.02 and subtracted from the logMAR value of the best line completely read. Contrarily, when letters were missed on a line, the number of the letters was multiplied by 0.02 and added to the acuity. The formula: $\operatorname{logMAR}$ acuity $=x-0.02 y$, where $x$ is the logMAR value of line completely read by the subject and $y$ is the number of letters read in the lower row. Applying this method to the example above (Snellen acuity $6 / 12^{+2}$ ), the logMAR value of the letters read is: $0.3-0.02(2)=0.26$. For this method, the lower the logMAR VA score, the better the VA and vice versa, therefore, 0.02 units are subtracted for each letter correctly read in the lower row.)

Each of these methods was applied to the unaided and aided acuity values of the subjects. Each paired set of data (unaided methods 1 and 2) (aided methods 1 and 2) were subsequently compared using the descriptive, correlation and regression statistics. Where applicable, the Bland and Altman method ${ }^{14}$ of clinical data analysis was used. The Bland and Altman method of analysis is a graphical and simple calculation method of comparing clinical measurements ${ }^{14}$. It is considered the most appropriate method for clinical comparison of data that is designed to measure the same parameters ${ }^{15}$. The results of the analyses were evaluated for similarities and differences and discussed in this article. The results for the left eyes are not presented in this article because they were similar to those of the right eyes.

\section{Results}

\section{Descriptive statistics}

Table 1 was created to highlight the various components of the VA values involved in this study. It shows the best five and the worst five (OD) VA values extracted from the total 140 VA data. The Snellen unaided and aided VAs are shown. The corresponding logMAR VA values (unaided, aided and their differences) for methods 1 and 2 are shown for the ten subjects. The table shows that, for method 1 , the logMAR values for the best VAs were higher than for the worst VAs. The contrary is the case for the method 2. The ranges and means for method 1 (aided and unaided) were lower than those of method 2, but their SD were the same. The improvement in VAs (unaided minus aided), their range, means and standard deviations (SD) are the same for both methods. 
Table 1: Showing the best five and worst five VA raw data (OD) in the study. The differences between the unaided and aided VA for the two methods are shown. The range, mean and SD values for the ten values are shown.

\begin{tabular}{|c|c|c|c|c|c|c|c|}
\hline \multicolumn{2}{|c|}{ SNELLEN } & \multicolumn{6}{|c|}{ LOGMAR } \\
\hline & & \multicolumn{3}{|c|}{ Method 1} & \multicolumn{3}{|c|}{ Method 2} \\
\hline Unaided & Aided & $\begin{array}{l}\text { Unaided } \\
\text { (UAOD1) }\end{array}$ & $\begin{array}{l}\text { Aided } \\
\text { (AOD1) }\end{array}$ & $\begin{array}{l}\text { Difference } \\
\text { (DOD1) }\end{array}$ & $\begin{array}{l}\text { Unaided } \\
\text { (UAOD2) }\end{array}$ & $\begin{array}{l}\text { Aided } \\
\text { (AOD2) }\end{array}$ & $\begin{array}{l}\text { Difference } \\
\text { (DOD2) }\end{array}$ \\
\hline $6 / 7.5^{-2}$ & $6 / 6^{-1}$ & 0.96 & 1.08 & 0.12 & 0.14 & 0.02 & 0.12 \\
\hline $6 / 12^{-1}$ & $6 / 12$ & 0.78 & 0.80 & 0.02 & 0.32 & 0.30 & 0.02 \\
\hline $6 / 15$ & $6 / 12$ & 0.70 & 0.80 & 0.10 & 0.40 & 0.30 & 0.10 \\
\hline $6 / 20^{+1}$ & $6 / 15^{+1}$ & 0.62 & 0.72 & 0.10 & 0.48 & 0.38 & 0.10 \\
\hline $6 / 20$ & $6 / 20^{+2}$ & 0.60 & 0.64 & 0.04 & 0.50 & 0.46 & 0.04 \\
\hline $6 / 48^{-1}$ & $6 / 30^{+2}$ & 0.18 & 0.44 & 0.26 & 0.92 & 0.66 & 0.26 \\
\hline $6 / 48^{-2}$ & $6 / 30$ & 0.16 & 0.40 & 0.24 & 0.94 & 0.70 & 0.24 \\
\hline $6 / 60^{+2}$ & $6 / 48^{-2}$ & 0.14 & 0.16 & 0.02 & 0.96 & 0.94 & 0.02 \\
\hline $6 / 60^{+1}$ & $6 / 48$ & 0.12 & 0.20 & 0.08 & 0.98 & 0.9 & 0.08 \\
\hline $6 / 60$ & $6 / 48^{+1}$ & 0.1 & 0.22 & 0.12 & 1.0 & 0.88 & 0.12 \\
\hline Range & & $0.1-0.96$ & $0.16-1.08$ & $0.02-0.26$ & $0.14-1.0$ & $0.02-0.94$ & $0.02-0.26$ \\
\hline Mean & & 0.436 & 0.546 & 0.110 & 0.664 & 0.554 & 0.110 \\
\hline SD & & 0.33 & 0.31 & 0.83 & 0.33 & 0.31 & 0.83 \\
\hline
\end{tabular}

Table 2 shows the results of the summary descriptive statistics for all the subjects. The range, mean and standard deviation values (SD) for the unaided, aided and differences using the two methods of data presentations are summarized in table 2 . The method 1 val- ues for range and mean for the unaided and aided VAs were lower than the method 2 values. The VA difference values (unaided minus aided) were, however, the same for both methods. All the standard deviations for both methods were the same. 
Table 2: Showing the ranges, means and SD for the logMAR VA values for all subjects. The analysis of the differences in VA between the unaided and aided (unaided minus aided) for method (DOD1) and method 2 (DOD2) are presented as 'difference' in the table. The range, mean and SD of differences of the methods were the same.

\begin{tabular}{|l|l|l|l|l|l|l|}
\hline \multirow{2}{*}{ Visual acuity } & \multicolumn{3}{l}{ Method 1 (logMAR) } & \multicolumn{2}{l|}{ Method 2 (logMAR) } \\
\cline { 2 - 7 } & Range & Mean & SD & Range & Mean & SD \\
\hline Unaided & $0.06-0.96$ & 0.39 & 0.15 & $0.14-1.04$ & 0.71 & 0.15 \\
\hline Aided & & & & & & \\
$($ AOD) & $0.10-0.8$ & 0.50 & 0.16 & $0.30-1.00$ & 0.60 & 0.16 \\
\hline Difference & $0.00-0.78$ & 0.13 & 0.10 & $0.00-0.78$ & 0.13 & 0.10 \\
\hline
\end{tabular}

\section{Regression analyses}

The scatter plots of the VA values for the unaide VA for the two methods (UAOD1 versus UAOD2) are shown in Figure 1. There is an inverse relationship between the logMAR VA of the two methods ( $r$ $=-1.00 ; p<0.05)$. The regression equation for scatter plot is $y=1.1-x$, where for method $1, y$ represents UAOD1 and $x$ represents UAOD2 and for method 2, $y$ represents UAOD2 and $x$ represents UAOD1 value.

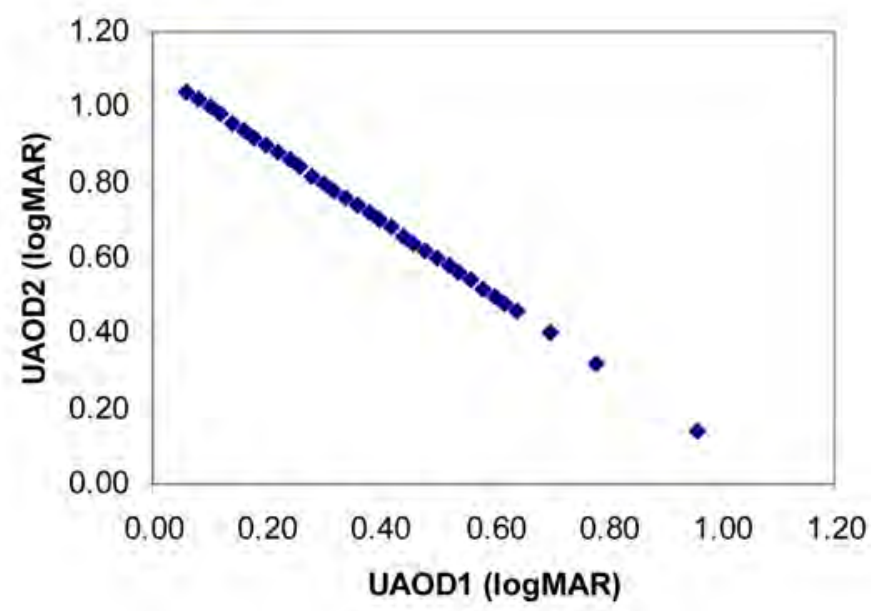

Figure 1. The scatter plots for UAOD1 plotted against UAOD2. The plot shows a perfect inverse relationship between methods 1 and 2 . All the 140 data points are not shown due to the fact that most of the coordinates are the same and the points therefore, lie on each other.

The scatter plots for the aided VAs (ADOD1 plotted against ADOD2) are shown in Figure 2. The regression line shows a perfect inverse relation between methods 1 and $2(r=-1, p<0.05)$. The regression equation for scatter plot is $y=1.1-x$, where for method 1, $y$ represents ADOD1 and $x$ represents ADOD2 and for method 2, $y$ represents ADOD2 and $x$ represents ADOD1.

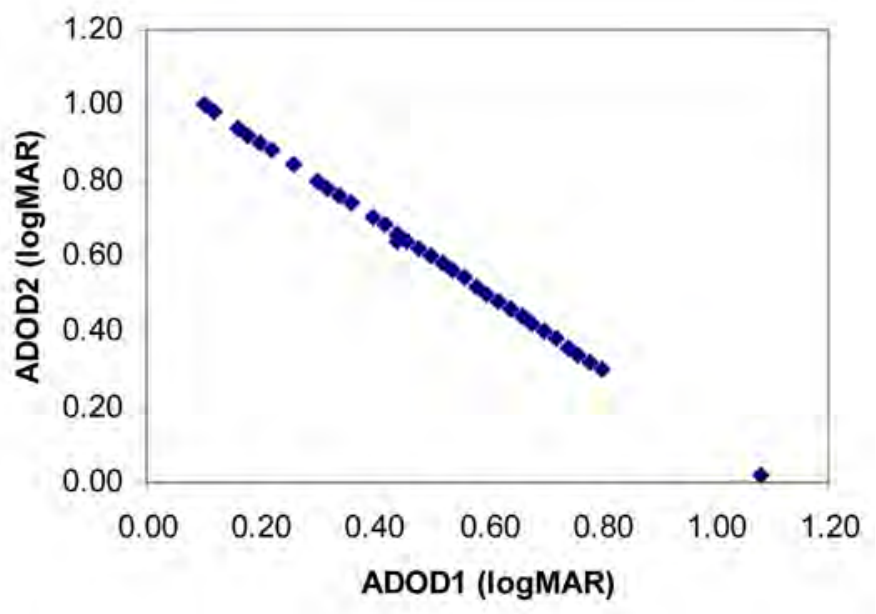

Figure 2. The scatter plots for aided VA, ADOD1 and ADOD2. The plot shows a perfect inverse relationship between methods 1 and 2. Again, most of the coordinates are the same; hence many points lie on each other.

A scatter plot of the differences between the unaided and aided VA using methods 1 and 2 is illustrated in Figure 3. The graph shows a positive correlation ( $r$ $=+1, p<0.05$ ), with regression equation $y=0.00+x$, where for method $1, y$ represents DOD1 and $x$ represents DOD2, while for method 2, $y$ represents DOD2 and $x$ represents DOD1. 


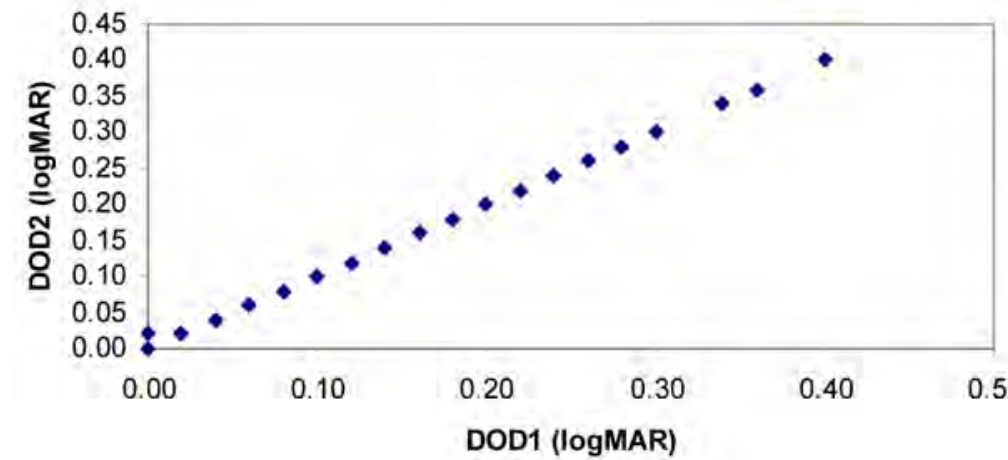

Figure 3. Showing the difference between the unaided VA and aided VA (method 1), plotted against the difference between unaided VA and aided VA (method 2) for the right eyes (UAOD1ADOD1 versus UAOD2 - ADOD2) or (DOD1 - DOD2). The graph shows a perfect positive correlation between the values.

\section{Bland and Altman analyses}

The Bland-Altman plot for the differences between the two methods, DOD1 and DOD2 shows that all the differences (DOD1 - DOD2) are zero, indicating a perfect agreement (Figure 4).

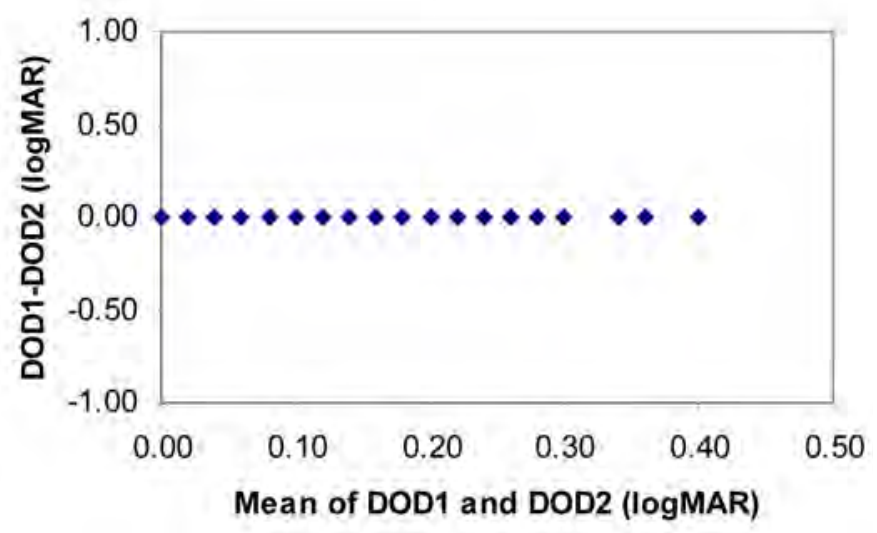

Figure 4. Showing the Bland and Altman plot for the differences (DOD1 and DOD2) of the unaided and aided logMAR values of the two methods. All the points align at 0.00 of the $y$-axis because all the differences (DOD1-DOD2) were zero. Also, the limit of agreement lines is not indicated because the differences are all zero.

\section{Discussion}

LogMAR VA measurements depend on chart design and scoring method used for the data collection ${ }^{16}$, therefore, when comparing findings between research studies, the type of chart and scoring methods should be taken into consideration. In view of these, there is a need for caution when comparing VA study find- ings. It has also been recommended that, to ensure comparison in VA values between studies and different study populations, it would be useful if clinical scientists worked to standardize VA testing protocols and report results in a manner consistent with the way in which they were measured ${ }^{9}$. Hazel and Elliot ${ }^{16}$ recommended that journals should be promoting a clear presentation of logMAR VA data. Doyle and Casswell ${ }^{17}$ have criticized Bhatnagar et al ${ }^{18}$ for presenting their VA data in several formats (number of acuity lines lost, number of letters lost and the reciprocal of $\log$ MAR), without mentioning the actual logMAR VA in the article. Although there are VA reports in the literature in which the methods of VA scoring were stated $^{10,11,19-21}$, there are others ${ }^{22-24}$ in which this was not indicated. It is difficult to compare VA data with existing values, if methods of collection or scoring are unknown. It is therefore, considered important for researchers to indicate the scoring methods of logMAR VA for future comparison. Even when the method of acuity scoring is known, it will be difficult to compare data, if the relationships between the two methods are not known. Findings in this study will fill this gap.

Correlation and linear regression methods are commonly used in the interpretation of quantitative method comparison studies ${ }^{25}$ and are most widely used by optometrists $^{26}$. These methods were combined with descriptive statistics and Bland and Altman analy$\mathrm{sis}^{14}$, all of which were considered to be relevant.

\section{Descriptive statistics}

The sample of ten subjects selected to highlight the various components of the VAs included in the study (Table 1), showed that, for method 1, the better Snellen VA values had higher logMAR values than the poor VA for both the unaided and aided VA values. The contrary is the case for method two. This has implications for statistics of a large number of data. If there are many subjects with good VAs (for example $6 / 5$ to 6/9) in a sample, then the range and mean VA values will be higher for method 1 than method 2, and vice versa. Also, Table 1 shows that the ranges and means of method 1 were lower than those of Method 2, the standard deviations were, however, the same. The ranges, means and SD for the difference (improvement in VA following ophthalmic compensation), DOD1 and DOD2 were the same for the two methods. 
The descriptive statistics (ranges, means and SD) for the entire subjects showed a similar trend with Table 1 for both methods 1 and 2 as shown in Table 2 . The reason for the differences between the two methods is that, method 1 measures the number of letters read, while method 2 measures the size of letter read. As shown in the introduction, if a subject read 6/60 lines only, this is recorded as $0.1 \log$ MAR for method 1 and 1.0 for method 2. Also, 6/12 will be 0.8 for method 1 and 0.3 for method 2. It becomes obvious that as the value of one method increases, the value of the other decreases and vice versa. These differences would vary the ranges and the means of the data, depending on the magnitudes of VA contained in the sample.

The standard deviations for the two methods, however, remained the same for all data because, recording a set of logMAR values using the two methods or when a set of Snellen acuity data are converted to logMAR using the two methods, the differences between the mean of the set of data and each individual data remain the same. Example, for a set of data: 6/60, $6 / 48,6 / 38$ and $6 / 30$, the logMAR values for method 1 are $0.1,0.2,0.3$, and 0.4 , while the logMAR values for method 2 are 1.0, 0.9, 0.8 and 0.7. The means for methods 1 and 2 are 0.25 and 0.85 respectively. Subtracting the individual logMAR values from the means, gives $0.15,0.05,-0.05$ and -0.15 respectively for method 1 and $-0.15,-0.05,0.05$ and 0.15 respectively for method 2. This explains why the standard deviations are the same for the two methods for all sets of data.

The range and mean values were consistently lower for method 1 than for method 2 because there were more poor VA values than good VA values in the sample. This is because the data were obtained from children with albinism, who generally have poor VA due to foveal hypoplasia as well as light fogging due to iris trans-illumination ${ }^{13}$. If more of the VAs were for example, in the regions of $6 / 7.5$ and $6 / 6$, then method 1 would have higher range and mean values than method 2.

The range, mean and standard deviation values of the differences between the unaided and aided VAs (improvement in vision) for the two methods were, however, the same (Tables 1 and 2) because, the values of the differences between the aided and unaided VA always remain the same irrespective of the meth- od employed. For example, suppose the unaided VA for a subject was 6/48 and the aided VA for the same subject was 6/30. The difference between the two VA values using method 1 is $0.4-0.2=0.2$; and using method 2, the difference is $0.9-0.7=0.2$. Similarly, for unaided VA of 6/12 and aided VA of 6/6, the difference using method 1 is $0.8-1.1=0.3$ and for method 2; it is $0.3-0.0=0.3$. This equality suggests that, although the range and means of unaided and aided VA values for a population sample cannot be compared if different methods of data scoring are used in different studies; the ranges, means and standard deviations of the improvement in vision following optical compensations (differences between unaided and aided VA) can be compared.

\section{Correlation and regression statistics}

The negative correlation $(r=-1)$ for the unaided and aided values for methods 1 and 2 (See Figures 1 and 2) was due to the inherent differences between the two procedures. A simple example can be used to corroborate this: For a set of VA: 6/60, 6/48, 6/38, $6 / 30$ and $6 / 24$, the logMAR VA values for method 1 are $0.1,0.2,0.3,0.4$ and 0.5 , but for method 2 the values are $1.0,0.9,0.8,0.7,0.6$ showing that as the values of one logMAR scoring method is increasing the other is decreasing. The perfect negative correlations indicate that, the value of method one can be used to predict the value of the other and vice versa. The differences between the unaided and aided VAs (improvement in VA) were positively correlated for the two methods $(r=+1, p<0.05)$ (Figures 3$)$ indicating that the regression equation of one value can be used to predict the other.

\section{Bland and Altman analysis}

This analysis establishes agreement between clinical data ${ }^{15}$ and therefore, was only applicable for differences between unaided VA values which were similar when the two methods were used. The Bland and Altman plot of the DOD1 - DOD2 against the means of DOD1 and DOD2 (Figure 4) shows a perfect agreement as the differences between the DOD1 and DOD2 were all zero. This supports the descriptive, correlation and regression analysis that the magnitude of improvement in VA values can be compared irrespective of whether method 1 or 2 is used in different studies. 


\section{Conclusion}

This study established that the two methods of data scoring described in this article should be used independently to analyze VA data, and that results of unaided or aided VA from either method should not be compared with those of the other. However, analyses or results of the improvement in vision obtained from one method can be compared to the other method. It is, therefore compulsory that whichever method used to present data in a study should be stated, so that those who might want to compare this with future findings would know and decide whether or not their data could be compared. Also, each method needs to be considered for its applicability to a specific study. In any comparative VA study, where there is a possibility of the subject missing some letters in the rows with large letters, while reading smaller rows, then method 1 must be used to score the acuity values. This is because this method quantifies the number of letters that are read. In situations where all the letters before the best acuity line are read, either method can be used. However, only the improvement in vision (for example following optical compensation or refractive surgery) results from one method can be compared with the other.

\section{Acknowledgement}

We acknowledge assistance of the following in the data analysis: Professor AKA Amey, Head of Statistics Department at the Venda University of Technology, Mrs. T. Esterhuizen, of the Nelson Mandela School of Medicine and Mr. M. Kobe, Discipline of Optometry, University of KwaZulu Natal. Also, we wish to thank Mr. HL Sithole for commenting on the manuscript.

\section{References}

1. Bailey IA, Lovie JE. New design principle for visual acuity letter charts. Am J Optom Physiol Opt 197653 740-745.

2. Ferris FL, Kassoff A, Bresnick GH, Bailey I. New visual acuity charts for clinical research. Am J Ophthalmol 1982 94 91-96.

3. Strong G, Woo G. A distance visual acuity chart incorporating some new design features. Arch Ophthalmol 1985103 44-46.

4. Al-Mufarrej MM, Abo-Hiemand FA, Oduntan AO. A new Arabic distance visual acuity chart. Optom Vis Sci 199673 59-61.
5. Oduntan AO, Briggs ST. An arabic letter distance visual acuity test chart for young children and illiterate adults. Ophthal Physiol Opt 199919 431-437.

6. Oduntan AO. Distance visual acuity test chart for young children and illiterate adults with low vision. S Afr Optom 200665 100-106.

7. Moseley MJ. Graphical representation of visual acuity data. Ophthal Physiol Opt 199717 441-442.

8. Lovie-Kitchin JE. Validity and variability of visual acuity measurements. Ophthal Physiol Opt 19888 363-370.

9. Moutray TN, Williams MA, Jackson JA. Change of visual acuity recording methods in clinical studies across the year. Ophthalmologica 2008222 173-177.

10. Roser DA, Laidlaw DAH, Murdoch IE. The development of a record logMAR visual acuity chart for use in routine clinical practice. Br J Ophthalmol 200185 432-436.

11. Vision in pre-schoolers (VIP) study group. Visual acuity results in school aged children and adults: Lea symbols chart versus Bailey-Lovie chart. Optom Vis Sci 200380 650-654.

12. Becker R, Teichler G, Graf M. Reproducibility of visual acuity assessment in normal and low visual acuity. Strab 200715 3-6.

13. Raliavhegwa M. Vision problems among children with oculocutaneous albinism attending special education schools in the Northern Province of South Africa. MOptom dissertation, University of the North, South Africa, 2001.

14. Bland J, Altman D. Statistical methods for assessing agreement between two methods of clinical measurement. Lancet 19861 307-310.

15. Hanneman SK. Design, analysis, and interpretation of method-comparison studies. AACN Adv Crit Care 19 223-

16. 234 .

Hazel CA, Elliot DB. The dependence of logMAR acuity on chart design and scoring rule. Optom Vis Sci 200279

17. 788-792.

Doyle E, Casswell AG. The presentation of data relating to

18. visual acuity. Eye 200721 284-285.

Bhatnagar A, Musadiq M, Yang YC. Late onset visual decline following successful treatment of subfoveal choroidal neovascularization with photodynamic therapy. Eye 2006

19. 20 491-493.

Laidlaw DAH, Abbott A, Rosser DA. Development of clinically feasible logMAR alternative to the Snellen chart:

20. performance of the compact reduced visual acuity chart in amblyopic children. Br J Ophthalmol 200387 1232-1234. Bourne RRA, Rosser DA, Sukudom P, Dineen B, Laidlaw DAH, Johnson GJ, Murdoch IE. Evaluating a new logMAR

21. chart designed to improve visual acuity assessment in population-based surveys. Eye 200317 754-758.

Tang W, Heng Wee-Jin, Lee Hung-Ming, Fam Han-Bor Lai Nai-Shin. Efficiency of measuring visual performance of

22. LASIK patients under photopic and mesopic conditions. Ann Acad Med Singapore 200635 541-546.

23. Ross WH, Stockl FA. Visual recovery after retinal detachment. Curr Opinion Ophthalmol 200011 191-194. 
24. Kadanosono K, Yabuki K, Nishide T, Uchio E, Marron JA. Measured visual acuity of fellow eyes as a prognostic factor in macular hole surgery. Am J Ophthalmol 2003135 4493-4498.

25. Alfonso JF, Fernandez-Vega, Valcare B, Montes-Mico R. Visual performance after AcrySofReSTOR aspheric intraocular lens implantation. J Optom 20081 30-35.

26. Armstrong RA, Eperjesi F, Gilmartin B. The use of correlation and regression methods in optometry. Clin Exp Optom 200588 81-88. 